\title{
Recombinant rabies virus expressing chimeric Omp31/sodC or AHCY gene from Brucella melitensis elicits high level of antibodies and secretary cytokines in mice
}

\section{Zhenguang Li ( $\sim$ lizhenguang@caas.cn )}

Institute of Special Economic Animal and Plant Sciences

Hongwei Zhu

Ludong University

Yanling Yang

Chinese Academy of Agricultural Sciences Institute of Special Animal and Plant Sciences

\section{Fengxue Wang}

Chinese Academy of Agricultural Sciences Institute of Special Animal and Plant Sciences

\section{Menghang Wang}

Chinese Academy of Agricultural Sciences Institute of Special Animal and Plant Sciences

\section{Na Sun}

Chinese Academy of Agricultural Sciences Institute of Special Animal and Plant Sciences

\section{Shipeng Cheng}

Chinese Academy of Agricultural Sciences Institute of Special Animal and Plant Sciences

\section{Zhen F. Fu}

University of Georgia

\section{Yongjun Wen}

Chinese Academy of Agricultural Sciences Institute of Special Animal and Plant Sciences

\section{Research article}

Keywords: Rabies virus; Brucella; Omp31/SodC gene; AHCY gene; Cellular and humoral immunity.

Posted Date: October 18th, 2019

DOI: https://doi.org/10.21203/rs.2.16210/v1

License: (c) (1) This work is licensed under a Creative Commons Attribution 4.0 International License. Read Full License 


\section{Abstract}

Background: The rabies virus (RV) vector LBNSE expressing foreign antigens has shown considerable promise as vaccines against viral and bacteria diseases, which is effective and safe. We produced a new RV-based vaccine vehicle expressing Brucella melitensis Omp31/sodC or AHCY gene by reverse genetics technology. The aim of this study was to investigate the cellular and humoral immunity of recombinant viruses. Results: The recombinant virus rLBNSE-SOD and rLBNSE-AHC retained growth properties similar to those of vector rLBNSE both in BSR and mNA cell culture. The Omp31/SODC and AHCY gene was expressed and detected by immunostaining. To compare the immunogenicity of LBNSE-SOD and LBNSEAHC, mice were immunized with each of recombinant virus by intramuscular (i.m.). Then mice were bled at days 7,14 after the immunization for the measurement of cytokines and virus neutralizing antibody (VNAs). The parent virus (LBNSE) without expression of any foreign molecules was included for comparison. It was found that mice inoculated with LBNSE-SOD and LBNSE-AHC showed no any signs of disease and exhibited seroconversion against RV. Our findings showed that mice were immunized with each of these recombinant RVs by intramuscular (i.m.) developed efficacy cellular and humoral immunity. The mRNA level of cytokines (IFN- $y$ and IL-2; IL-4 and IL-10) and VNA level against rabies virus in the blood of mice were increasing after immunization with recombinant RVs. There were no obvious histopathological changes in the brain samples of all mice. Conclusions: The studies suggested that recombinant RVs expressing Omp31/SodC or AHCY gene would elicit high level of antibodies and secretary cytokines and provided a promising vaccine candidate in mice.

\section{Background}

Brucellosis, caused by Brucella spp., is a worldwide spread zoonotic disease transmitted from domestic animals to humans. Brucella causes abortions and sterility in infected domestic animals and various insidious syndromes in humans. The disease is a major cause of direct economic losses and is an impediment to trade and export [1]. Identifying novel Brucella antigens that can elicit a protective immune response, without the drawbacks of live attenuated vaccines, could enable development of nextgeneration vaccines. Previous study showed S-adenosyl-L-homocysteine hydrolase (AdoHcyase, AHCY) was identified and expressed in Escherichia coli. The recombinant AHCY induced a strong antibody response and a high level of protection against $B$. melitensis $16 \mathrm{M}$ challenge, which indicated that rAdoHcyase could be a useful candidate for the development of subunit vaccines against $B$. Melitensis [2]. In previous study, intramuscular injection with a plasmid DNA carrying Omp31/SodC gene (pcDNAOmp31/SodC) was able to induce a protective immune response [3]. And intraspleen immunization with pcDNA- Omp31/SodC vaccine efficiently induced Omp31/SodC -specific CD4+ and CD8+ T cells [4]. Recently, an improved recombinant Brucella abortus vaccine strain RB51SOD by over-expressing Cu-Zn superoxide dismutase (SOD), has been shown to confer better protection than parental strain RB51 in a mouse model, which indicated that SOD could be a new immunogenic candidate gene for the development of subunit vaccines against $B$. Melitensis [5]. Oral immunization of mice with recombinant 
Lactococcus lactis expressing SOD gene of Brucella abortus triggers protective immunity against $B$. abortus infections [6].

The reverse genetics system has developed rapidly in recent years, which is helpful to create genetically engineered vaccine $[7,8]$. Recombinant rabies virus vaccine expressing proteins of other pathogens stably show powerful protection against other infectious diseases as vaccine vector[9-11], while the safety concerns of recombinant rabies virus vaccine have previously been stated in our study [12]. In previous reports, it was indicated that recombinant RV expressing chemokines/cytokines including granulocyte-macrophage colonystimulating factor (GM-CSF), macrophage-derived chemokine (MDC), and macrophage inflammatory protein (MIP-1a), can recruit and/or activate more DCs to enhance the immunogenicity[12, 13].

In present study, we generated a recombinant RV attenuated vaccine strain expressing Brucella abortus Omp31/SodC (LBNSE-SOD) or AHCY gene (LBNSE-AHC) which is immunogenic candidate gene and plays an important role in inducing the protective immunity as well. In order to further investigate the immunological characteristics of the recombinant RV, forty 5-week-old female mice were experimentally immunized with LBNSE-SOD and LBNSE-AHC. And hematocytes analysis, pathological changes, humoral and cell-mediated immune responses were evaluated in this study.

\section{Results}

\section{Construction and rescue of infectious chimeric clone and detection of rRABV expressing Brucella Omp31/SodC or AHCY gene}

To construct recombinant rabies virus (RV) expressing Brucella Omp31/SodC or AHCY gene, a recombinant virus was developed using the RV infectious CDNA clone pLBNSE as the backbone as described in Methods. Infectious clone was shown in Figure 1A, which Brucella Omp31/SodC or AHCY gene was inserted into pLBNSE between the $G$ and $L$ genes by $B$ siwl and Nhel. The rescued chimeric clone was named as LBNSE-SOD and LBNSE-AHC confirmed by IFA and DNA sequencing. Several nucleotide changes including foreign genes inserted restriction endonuclease (RE) sites were observed throughout the genome.

To detect the rescued infectious clone from the cell culture supernatant, the transfected cells were fixed and examined by an indirect fluorescence assay (IFA). It was indicated that the BSR cells transfected with LBNSE (Fig. 2B), LBNSE-SOD (Fig. 2C) and LBNSE-AHC (Fig. 2D) showed green fluorescence, respectively. However, control cells remained negative (Fig.2A). Full-genome sequencing results showed that recovered virus LBNSE-SOD and LBNSE-AHC maintained the nucleotide sequence of the original plasmids pLBNSEOmp31/SodC and pLBNSE-AHCY.

\section{Growth curve of recombinant RVs}


One concern about a recombinant RV expressing Brucella Omp31/SodC or AHCY gene was whether this will affect the viral replication cycle, resulting in a recombinant RV that grows slowly or at low titer. To study the replication kinetics of LBNSE-SOD and LBNSE-AHC, a multicycle growth curve was performed by infecting BSR cells at an $\mathrm{MOI}$ of 0.01 . From the Figure 1, we can get the information of growth kinetics of the reconstituted viruses and the parental virus. The skeleton virus LBNSE and the chimeric virus LBNSE-SOD and LBNSE-AHC showed the similar growth kinetics in BSR cells (Figure 1B) and mNA cells (Figure 1C). The recombinant RV grew very similarly and reached approximately the same titer at similar time points, indicating there were no differences in viral spread. Therefore, the insertion of Omp31/SodC and AHCY genes did not have any major impact on the viral life cycle. The cloned virus was further amplified by subsequent passages in BSR cells. And the titer of passage- 3 rescued virus was determined.

\section{Cells count in the heparinized blood of mice}

To determine the effect of recombinant rabies viruses expressing Brucella genes on hematocyte differentiation, mice were immunized with each of rRV virus and heparinized blood were harvested and hematocytes were auto-counted by VetScan HM5 (Gurnee, IL). As showed in Fig. 3A and 3B, there are no significant different change of total white blood cells and red blood cells at different time-points between each group. Fig. $3 \mathrm{C}$ showed that the number of platelets started to increase at $7 \mathrm{dpi}$ and significantly higher than control group in mice immunized with LBNSE, LBNSE-SOD and LBNSE-AHC $(p<0.05)$. In Fig. $3 D$, the number of lymphocytes also started to significantly increase at $7 \mathrm{dpi}$ than control group in mice immunized with LBNSE and LBNSE-AHC, while significantly higher than control group in LBNSE-SOD and LBNSE-AHC groups by $14 \mathrm{dpi}(p<0.05)$, which was going down at $14 \mathrm{dpi}$ in LBNSE group. As showed in Fig. 3E, ONLY LBNSE group showed significantly more number of monocytes than other groups $(p<$ $0.05)$, and there are no significant different change in other groups through the experiment course. Finally, in Fig. 3F, ONLY LBNSE-AHC group showed significantly more number of neutrophils than other groups ( $p$ $<0.05)$, and there are no significant different change in other groups through the experiment course.

\section{The mRNA level of cytokines in the heparinized blood of mice}

To address the cellular and humoral immunity after immunization with recombinant rabies viruses expressing Brucella genes, mice were immunized with each of $\mathrm{rRV}$ virus and heparinized blood were harvested and mRNA level of cytokines (IFN- $\gamma$ and IL-2; IL-4 and IL-10) in the blood were measured by real-time PCR. Fig. 4A and 4B showed mRNA level of IL-2 and IFN- $\gamma$ increased significantly at $7 \mathrm{dpi}$ and reached the highest levels by $14 \mathrm{dpi}$ in the LBNSE-SOD and LBNSE-AHC groups, compared to control group $(p<0.05)$. And LBNSE group showed significantly more mRNA level of IL-2 and IFN- $\gamma$ at $7 \mathrm{dpi}$. As shown in Fig. 4C and 4D, mRNA level of IL-4 started to increase while IL-10 peaked at $7 \mathrm{dpi}(p<0.01)$. However IL-10 started to decline by $14 \mathrm{dpi}$ in all immunized groups, while there is still significantly more mRNA level than control group. Of interest, immunization with LBNSE-SOD and LBNSE-AHC were able to express relatively more IL-10 levels in the sera than with parental virus. On the other hand, delivery with LBNSE-SOD could develop relatively more IL-4 levels in the blood than with LBNSE-AHC and parental virus. 


\section{The ELISA level of cytokines in the sera of mice}

To confirm the expression of Th1 and Th2 cells in the sera after immunization with recombinant rabies viruses expressing Brucella genes, ELISA level of cytokines (IFN-y and IL-10) in the sera of mice were detected by mouse quantitative ELISA Kit according to the manufacturer's instructions. Fig. 5A showed ELISA level of IFN-y increased significantly at $7 \mathrm{dpi}$ and reached the highest levels by $14 \mathrm{dpi}$ in all the vaccinated groups, compared to control group $(p<0.05)$. As shown in Fig. 5B, ELISA level of IL-10 peaked at $7 \mathrm{dpi}$ and started to decline by $14 \mathrm{dpi}$ in all immunized groups $(p<0.05)$, while there is no significant different change through the experiment in control group. Of note, immunization with LBNSE-SOD and LBNSE-AHC can express relatively more IFN- $y$ and IL-10 levels in the sera than with parental virus.

\section{The VNA level against rabies virus in the sera of mice}

In order to address the VNAs production against rabies virus after recombinant RV immunization $₫$ blood samples were collected at 7, $14 \mathrm{dpi}$, and sera VNAs were determined by RFFIT. As shown in Fig. 6, VNA titres in sera increased significantly at $7 \mathrm{dpi}$ and reached the highest levels by $14 \mathrm{dpi}$ in the LBNSE, LBNSE-SOD and LBNSE-AHC groups, compared to control group. While there were no significant different VNA titres between in mice immunized with recombinant RVs and the parent virus $(p>0.05)$.

\section{Gross pathological and histopathological changes}

To investigate the effects of recombinant rabies viruses expressing Brucella Omp31/SodC or AHCY gene on tissues pathological and inflammatory cell infiltration in brains. Mice were immunized with each of recombinant rabies virus and brains were collected and stained with H.E. No obvious pathological lesions were found in all organs of control mice treated with Medium and LBNSE group at necropsy, while 1 out of 10 mice in LBNSE-SOD and LBNSE-AHC group showed mild hemorrhage and swelling in lymph nodes (data not shown). Under microscopic examination from Fig.7, no perivascular-cuffing and neuronal degeneration showed in all brain samples of control and immunized groups. There is no inflammatory cell infiltration in the brains of control and LBNSE groups. While only one out of ten mice immunized with LBNSE-SOD and LBNSE-AHC showed mild inflammatory cell infiltration in brain. vascular cuff vascular cuff

\section{Discussion}

Our previous studies have shown that recombinant RABV expressing dendritic cell-activating molecules such as GM-CSF, MDC, MIP-1a and bacterial flagellin could enhance the innate and adaptive immune response to vaccination and led to better protection than with the parent virus when challenged with virulent $R A B V$ in mice[12, 13, 22, 23]. It is confirmed that recombinant rabies virus vaccine strain-based vectors expressing foreign genes or proteins provided the promising safe and effective vaccine candidates in mouse model. In this study, Brucella Omp31/SodC or AHCY gene was cloned into the pLBNSE genome respectively and recombinant virus LBNSE-SOD and LBNSE-AHC were rescued. After incorporation of Omp31/SodC and AHCY gene, the recombinant virus still grew to a high final titer of 
about $10^{7.0} \mathrm{FFU} / \mathrm{mL}$. From the multicycle growth curve, it showed that inset of Omp31/SodC and AHCY gene did not affect the growth and replication of recombinant rabies virus. Safety is a major concern for the use of live viral vector. However, this RV based vectors expressing Omp31/SodC and AHCY gene were completely safe in mice and apathogenic after intramuscular infection. For gross pathological, there was no obvious pathological lesion in all organs from almost all mice of LBNSE-SOD and LBNSE-AHC groups at necropsy (data not shown).

From hematocytes analysis results, there were the similar change tendency for different blood cells between LBNSE-SOD and LBNSE-AHC and parental virus. Except that ONLY LBNSE group showed significantly more number of monocytes than other groups and ONLY LBNSE-AHC group showed significantly more number of neutrophils than other groups. In previous study, T-cell proliferative responses and increased production of gamma interferon were also observed upon splenocyte restimulation with recombinant SOD and the pCDNA-SOD induced improved protection against challenge with the virulent strain B. abortus in BALB/c mice[24]. A liposome based DNA vaccine co-expressing SOD gene along with interleukin-18 (IL-18) was evaluated in experimental murine brucellosis. Higher lymphoproliferative response and IFN-gamma production were developed after treatment with recombinant DNA vaccine [25]. A recombinant DNA vaccine encoding SOD confered high protection against Brucella abortus 2308 by inducing specific CTL responses in mouse model[26]. In our study, the results of mRNA level of cytokines (IFN- $y$ and IL-2; IL-4 and IL-10) in the blood indicated that all cytokines except IL-10 showed continuous increasing by $14 \mathrm{dpi}$. The change trend of ELISA level of cytokines (IFN- $\gamma$ and IL-10) in the sera showed in Figure 5 was consistent with the results of mRNA level in blood showed in Figure 4. High VNA titers were detected in mice immunized with LBNSE-SOD and LBNSE-AHC and parental virus, there were no significant difference between recombinant RVs and parental virus. These data predict that treatment with LBNSE-SOD and LBNSE-AHC also induced high adaptive immunity as parental virus. Further studies are needed to address if recombinant RVs is effective to complete protect mice against rabies virus challenge.

In conclusion, the results presented here suggest that recombinant virus delivering Omp31/SodC and $\mathrm{AHCY}$ gene induced good immune response against rabies virus. The large insert genes from Brucella melitensis can be efficiently expressed by RV-based backbone, which further indicates their potential function as efficacious antiviral vaccines. Our results of immune response following rLBNSE which expresses Omp31/SodC or AHCY gene from Brucella melitensis imply further researches on subunit vaccine immunizations in mice, especially in order to reduce the risks of the virulence reversion of attenuated strain in the field application.

\section{Conclusions}

A new recombinant rabies virus expressing Brucella melitensis Omp31/sodC or AHCY gene was constructed in this study. The LBNSE- SOD and LBNSE-AHC showed the similar viral growth kinetics to parental virus both in BSR and mNA cell culture. Likewise, our findings show the expression of Omp31/sodC or AHCY gene in RABV results in increased cytokines secretary and VNA production. While 
there were no obvious histopathological changes and inflammatory cell infiltration in brains of mice after immunized with recombinant rabies virus. It is suggested that recombinant RVs expressing Omp31/sodC or AHCY gene are more immunogenic and provide a promising vaccine candidate in mice. However, the immune response of expressed $\mathrm{Omp31/sodC}$ or $\mathrm{AHCY}$ gene is warranted to address in the further research.

\section{Methods}

\section{Cells and viruses}

Mouse neuroblastoma cells (NA) were maintained in RPMI 1640 medium (Mediatech, Herndon, VA) supplemented with $10 \%$ fetal bovine serum (FBS) (Gibco, Grand Island, NY). BSR cells, a cloned cell line derived from BHK-21 cells, were maintained in Dulbecco's modified Eagle's medium (Mediatech) containing 10\% FBS. Recombinant RV strains were propagated in BSR cells. Fluorescein isothiocyanate (FITC)-conjugated antibody against the RV N protein was purchased from FujiRab (Melvin, PA). Challenge virus standard 11 (CVS-11) was propagated in NA cells.

\section{Plasmid construction}

The recombinant rabies virus plasmids, pLBNSE, were constructed as described previously [12]. Briefly, the skeleton plasmid pLBNSE was developed from an SAD L16 cDNA clone in pcDNA3.1 (+) (Invitrogen, Carlsbad, CA). And the transcription unit with the Bwsil and Nhel restriction sites was designed between the $G$ and $L$ genes by removing the pseudogenes. To produce a new rabies virus vector expressing Brucella Omp31/SodC and AHCY gene, the genes were amplified from by using Pfu polymerase (Biolabs, Inc.). And the primers used for the construction of these genes were designed by using Primer5.0. The PCR product was digested with BsIWI-Nhel and linked to the BsIWI-Nhel-digested plasmid pLBNSE. This plasmid was named as pLBNSE-SOD and pLBNSE-AHC (Fig. 1a) and the insert gene was confirmed by restriction analysis and DNA sequencing.

\section{Recombinant viruses rescue and insert genes expressing detection}

The full-length recombinant cDNA clones were purified and were co-transfected with the plasmids encoding the RV N, P, G, and L proteins into BSR cells which stably expressing T7 RNA polymerase [14] by standard methods [10], and infection was examined in 2 from 6 wells of co-transfected cells. The recombinant virus was rescued as described previously $[15,16]$. Briefly, BSR cells were transfected with $2.0 \mu \mathrm{g}$ of the full-length infectious clone, $0.5 \mu \mathrm{g}$ of $\mathrm{N}-, 0.25 \mu \mathrm{g}$ of $\mathrm{P}-, 0.1 \mu \mathrm{g}$ of $\mathrm{L}-$, and $0.15 \mu \mathrm{g}$ of Gexpressing plasmids using SuperFect Transfection Reagent (Qiagen, Valencia, CA) according to the manufacture's instruction. After 4 days, the culture medium was discarded and another fresh medium was added. And the culture supernatant was harvested after another 3 days, then the rescued recombinant viruses were examined by an indirect fluorescence assay using FITC-conjugated antibody against the RV N protein (Fujirebio Diagnostic, Inc. DA, USA). Rescued recombinant viruses were designated as LBNSE-SOD and LBNSE-AHC. 
To verify the insert genes, the Brucella Omp31/SodC gene was amplified by using primers 5'-

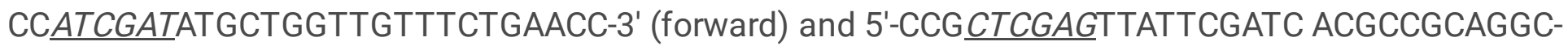
3 ' (reverse), while the Brucella AHCY gene was amplified by using primers 5'CCATCGATATGCAATTTTGCCTTCAG-3'(forward) and 5'-CCG CTCGAGTTAATACCTGTAG TGTTCCG$3^{\prime}$ (reverse). PCR products were purified using QIAquick gel extraction kit (Qiagen, Germantown, MD) and cloned into the pCR-Blunt II vector (Invitrogen). The amplified Omp31/SodC and AHCY genes were inserted between the Cla I and Xhol sites in the plasmid pCAGGS and were designated as pCAOmp31/SodC and pCA-AHCY, respectively. Then BSR cells were transfected with pCA-Omp31/SodC and pCA-AHCY using lipofectamine ${ }^{T M} 2000$ (Invitrogen) according to the manufacture's protocol. The transfected cells were fixed and examined by an indirect fluorescence assay (IFA), which using sera from mice against LBNSE-SOD or LBNSE-AHC as first antibody and Dylight 594-conjugated second antibody against the mouse IgG.

\section{The growth curve of recombinant virus}

BSR cells were plated in 60-mm-diameter dishes. After $16 \mathrm{~h}$ incubation, the cells were infected at an $\mathrm{MOI}$ of 0.01 (multicycle growth) with LBNSE, LBNSE-SOD and LBNSE-AHC. After incubation at $37^{\circ} \mathrm{C}$ for $1 \mathrm{~h}$, inocula were removed, and cells were washed three times with PBS to remove any unabsorbed virus. Three milliliters of complete medium was added back, and $100 \mu \mathrm{l}$ of tissue culture supernatants was removed at the indicated time points after infection. Titers of virus aliquots were determined in duplicate on BSR cells.

\section{Vaccination experiments in mice}

Forty 5-week-old female mice, whose serology is negative to Brucella melitensis and rabies virus, were purchased from Changchun Institute of Biological Products Co., Ltd. and raised by isolated cages in the Animal Facility, Division of Zoonosis, Institute of Special Economic Animal and Plant Sciences. Mice were randomly divided into 4 groups, and 10 mice in each group were used to verify the vaccination efficacy. Three groups of mice were vaccinated by i.m.route (biceps femoris muscle of right hindlimb) at 6 week of age with $10^{6}$ PFU of LBNSE, LBNSE-SOD, LBNSE-AHC with $100 \mu$ per dose, respectively. One group of mice inoculated i.m. with DMEM as a sham-treated control during the experiment. At days $0,7,14$ after immunization, heparinized blood was harvested and sera were collected from anesthetized mice for realtime PCR and serology test. At the end of experiment, all the mice were anesthetized and euthanized in a CO chamber.

The animal experiments herein described have been reviewed and approved by the local Animal Care \& Use Committee of the Institute of Special Wild Economic Animal and Plant Sciences, Chinese Academy of Agricultural Sciences, Jilin, China animal (welfare assurance number: SEAPS-IACUC-2015-004).

\section{Hematocytes counts in the blood}


Whole blood from each group were collected with EDTA for hematocyte count at day 0 , and at day 7, 14 after immunization, including WBC (white blood cell), RBC (red blood cell), platelet, lymphocyte, monocyte and neutrophils. Blood cells were counted using an automated cell counter VetScan HM5 (Gurnee, IL) according to the protocol.

\section{Quantitative real-time PCR (qRT-PCR)}

In order to measure the expression of cytokines in the blood, a quantitative real-time PCR assay was conducted in a BIO-RAD T100 ${ }^{\text {TM }}$ Thermal Cycler (Hercules, CA) as described previously [17-19]. Briefly, total RNA was extracted from blood samples according to the Qiagen RNeasy kit (Qiagen, Redwood, CA) and treated with DNase (Qiagen). The reverse transcriptase and DNA polymerase were used from a TransScript ${ }^{\mathrm{TM}}$ Green One-Step qRT-PCR SuperMix kit. Each PCR reaction ran in duplicate with $100 \mathrm{ng}$ DNase-treated RNA and $5 \mathrm{nM}$ each primer pairs. Amplification was operated at $50^{\circ} \mathrm{C}$ for $2 \mathrm{~min}$ and $95^{\circ} \mathrm{C}$ or $10 \mathrm{~min}$, followed by 40 cycles in two steps: $95^{\circ} \mathrm{C}$ for $15 \mathrm{~s}$ and $60^{\circ} \mathrm{C}$ for $1 \mathrm{~min}$. The copy numbers of

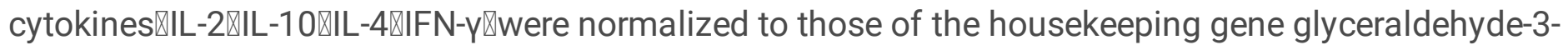
phosphate dehydrogenase (GAPDH). Levels of cytokines expression in samples of mice vaccinated with LBNSE and LBNSE-SOD, LBNSE-AHC are presented as the fold change over that detected in control group.

\section{Total IgG ELISA assay}

In order to confirm the level of cellular and humoral immunity, ELISA levels of IL-10 and IFN- $\gamma$ in the sera were detected by Mouse Quantitative ELISA Kit (Cat:M1000B and Cat:MIF00, R\&D Systems, Inc. Minneapolis, MN) according to the kit's protocol. The optical density was examined at $450 \mathrm{~nm}$ using a spectrophotometer (BioTek Instruments, VT). Calculation of IL-10 and IFN- $\gamma$ concentration was developed by linear regression analysis using software KC4 Signature Ver. 3.4 (Bio-Tek Instruments). A standard curve was made using known concentrations of mouse IL-10 and IFN-y provided in the ELISA Kit (R\&D Systems). The mouse IL-10 and IFN- $\gamma$ concentrations of samples from vaccinated and control groups were determined according to the standard curve using a 4-parameter logistic regression equation of the $\mathrm{KC} 4$ program. The results were presented as $\mathrm{pg} / \mathrm{L}$ for each sample.

\section{Rapid fluorescent focus inhibition test (RFFIT)}

RV neutralizing antibodies (VNAs) were detected using the rapid fluorescent focus inhibition test (RFFIT) as described previously [20], and titers of VNAs were normalized to international units (IU/ml). Briefly, $50 \mu$ of serial five-fold dilutions of serum were added in Lab-Tek Chamber slides (Nalge Nunc International, Rochester, NY). 50\% Fluorescing Foci dose (Fifty FFD50) of challenge virus standard (CVS11) was added to each chamber and incubated for $90 \mathrm{~min}$ at $37^{\circ} \mathrm{C}$. NA cells $\left(10^{5} \mathrm{cells}\right)$ were added into each chamber and the slides were incubated at $37^{\circ} \mathrm{C}$ for 20 hours. Then the cells were fixed and stained with FITC-conjugated anti-RABV $\mathrm{N}$ antibodies. After washing with PBS, twenty fields in each chamber were observed under a fluorescent microscope. Then the $50 \%$ endpoint titers were determined following the Reed-Muench formula. Then the titers of VNAs were normalized to international units (IU/ml). 


\section{Histopathology observation}

At necropsy, the tissue samples from brains were harvested and fixed in 10\% neutral-buffered formalin for histopathology observation following hematoxylin and eosin (H\&E) staining as described previously [21].

\section{Statistical analysis}

The statistical significance of the differences between various groups was analyzed by Graphpad Prism 5 v. 5.01 (Graphpad Software Inc., La Jolla, CA, USA). Student's t-test or one-way ANOVA was used to evaluate the significant difference. Statistical significance was set at $p<0.05$ and extremely significance at $p<0.01$.

\section{Abbreviations}

RV: Rabies virus; i.m: intramuscular; VNA: virus neutralizing antibody; rLBNSE: recombinant virus LBNSE; rRABV: recombinant rabies viruses; AHCY: S-adenosyl-L-homocysteine hydrolase; SOD: superoxide dismutase; NA: Mouse neuroblastoma cells; FBS: fetal bovine serum; CVS-11: Challenge virus standard 11; WBC: white blood cell; RBC: red blood cell; H\&E: hematoxylin and eosin; RE: restriction endonuclease.

\section{Declarations}

Ethics approval and Consent to Participate

The animal experiments herein described have been reviewed and approved by the local Animal Care \& Use Committee of the Institute of Special Wild Economic Animal and Plant Sciences, Chinese Academy of Agricultural Sciences, Jilin, China animal (welfare assurance number: SEAPS-IACUC-2015-004). And we have followed the 3 Rs guiding principles during the course of animal experiment.

Availability of data and materials

The data sets during and/or analyzed during the current study available from the corresponding author on reasonable request.

\section{Competing interests}

The authors have agreed that there is no conflict of interest between them.

Consent for publication

Not applicable.

\section{Funding}


The study was financially supported by the grant of National Natural Science Foundation of China (NSFC)'Molecular mechanism for synaptic recycling dysfunction induced by G protein of street rabies virus in primary mouse neural cells'(31572505), the National Program for Key Research Projects of China (2016YFD0500900 and 2017YFD0500900) and "311" program of Taizhou city. The funder Yongjun Wen and Zhenguang Li designed the study and wrote the article.

\section{Authors' contributions}

ZL and $\mathrm{HZ}$ conceived and designed the experiments. YY, FW, MW and NS performed the experiments. ZL and $\mathrm{SC}$ analyzed the data. $\mathrm{ZL}, \mathrm{HZ}, \mathrm{ZF}$, and $\mathrm{YW}$ wrote the article. All authors read and approved the final manuscript.

\section{Acknowledgements}

We thank all of the technicians who were involved in the animal care and sample collection. And we are grateful of Dr.Wenzhi Xue for many helpful suggestions and his critical reading of the manuscript.

\section{Author details}

astate Key Laboratory for Molecular Biology of Special Economic Animals, Institute of Special Economic Animals and Plants, Chinese Academy of Agricultural Sciences CAAS, Changchun, Jilin 130112, China.

bSinovet (Jiangsu) Biopharm. Co., Ltd., Taizhou, Jiangsu 225300, China. 'S Chool of Life Sciences, Ludong University, Yantai, Shandong 264025, China. ${ }^{\mathrm{d} C o l l e g e ~ o f ~ V e t e r i n a r y ~ M e d i c i n e, ~ K e y ~ L a b o r a t o r y ~ f o r ~ C l i n i c a l ~}$ Diagnosis and Treatment of Animal Diseases of Ministry of Agriculture, Inner Mongolia Agricultural University, Hohhot, Inner Mongolia 010018,China. ${ }^{~}$ Department of Pathology, University of Georgia, Athens, GA 30602, USA

\section{References}

[1] Seleem M.N., Boyle S.M., Sriranganathan N., Brucellosis: a reemerging zoonosis, Veterinary microbiology. (2010) 140:392-398.

[2] Yang Y., Yin J., Guo D., Lang X., Wang X., Immunization of mice with recombinant S-adenosyl-Lhomocysteine hydrolase protein confers protection against Brucella melitensis infection, FEMS immunology and medical microbiology. (2011) 61:159-167.

[3] Onate A.A., Cespedes S., Cabrera A., Rivers R., Gonzalez A., Munoz C., Folch H., Andrews E., A DNA vaccine encoding $\mathrm{Cu}, \mathrm{Zn}$ superoxide dismutase of Brucella abortus induces protective immunity in BALB/c mice, Infection and immunity. (2003) 71:4857-4861.

[4] Munoz-Montesino C., Andrews E., Rivers R., Gonzalez-Smith A., Moraga-Cid G., Folch H., Cespedes S., Onate A.A., Intraspleen delivery of a DNA vaccine coding for superoxide dismutase (SOD) of Brucella 
abortus induces SOD-specific CD4+ and CD8+ T cells, Infection and immunity. (2004) 72:2081-2087.

[5] Surendran N., Hiltbold E.M., Heid B., Sriranganathan N., Boyle S.M., Zimmerman K.L., Makris M.R., Witonsky S.G., Live Brucella abortus rough vaccine strain RB51 stimulates enhanced innate immune response in vitro compared to rough vaccine strain RB51SOD and virulent smooth strain 2308 in murine bone marrow-derived dendritic cells, Veterinary microbiology. (2011) 147:75-82.

[6] Saez D., Fernandez P., Rivera A., Andrews E., Onate A., Oral immunization of mice with recombinant Lactococcus lactis expressing $\mathrm{Cu}, \mathrm{Zn}$ superoxide dismutase of Brucella abortus triggers protective immunity, Vaccine. (2012) 30:1283-1290.

[7] Nagai Y., Paramyxovirus replication and pathogenesis. Reverse genetics transforms understanding, Rev Med Virol. (1999) 9:83-99.

[8] Billeter M.A., Naim H.Y., Udem S.A., Reverse genetics of measles virus and resulting multivalent recombinant vaccines: applications of recombinant measles viruses, Curr Top Microbiol Immunol. (2009) 329:129-162.

[9] McGettigan J.P., Foley H.D., Belyakov I.M., Berzofsky J.A., Pomerantz R.J., Schnell M.J., Rabies virus-based vectors expressing human immunodeficiency virus type 1 (HIV-1) envelope protein induce a strong, cross-reactive cytotoxic T-lymphocyte response against envelope proteins from different HIV-1 isolates, J Virol. (2001) 75:4430-4434.

[10] McGettigan J.P., Sarma S., Orenstein J.M., Pomerantz R.J., Schnell M.J., Expression and immunogenicity of human immunodeficiency virus type $1 \mathrm{Gag}$ expressed by a replication-competent rhabdovirus-based vaccine vector, J Virol. (2001) 75:8724-8732.

[11] Siler C.A., McGettigan J.P., Dietzschold B., Herrine S.K., Dubuisson J., Pomerantz R.J., Schnell M.J., Live and killed rhabdovirus-based vectors as potential hepatitis $C$ vaccines. Virology 292:24-34. (2001).

[12] Wen Y., Wang H., Wu H., Yang F., Tripp R.A., Hogan R.J., Fu Z.F., Rabies virus expressing dendritic cell-activating molecules enhances the innate and adaptive immune response to vaccination, Journal of virology. (2011) 85:1634-1644.

[13] Zhou M., Zhang G., Ren G., Gnanadurai C.W., Li Z., Chai Q., Yang Y., Leyson C.M., Wu W., Cui M., Fu Z.F., Recombinant rabies viruses expressing GM-CSF or flagellin are effective vaccines for both intramuscular and oral immunizations, PloS one. (2013) 8:e63384.

[14] Buchholz U.J., Finke S., Conzelmann K.K., Generation of bovine respiratory syncytial virus (BRSV) from CDNA: BRSV NS2 is not essential for virus replication in tissue culture, and the human RSV leader region acts as a functional BRSV genome promoter, J Virol. (1999) 73:251-259.

[15] Inoue K., Shoji Y., Kurane I., lijima T., Sakai T., Morimoto K., An improved method for recovering rabies virus from cloned cDNA, J Virol Methods. (2003) 107:229-236. 
[16] Takayama-Ito M., Inoue K., Shoji Y., Inoue S., lijima T., Sakai T., Kurane I., Morimoto K., A highly attenuated rabies virus HEP-Flury strain reverts to virulent by single amino acid substitution to arginine at position 333 in glycoprotein, Virus Res. (2006) 119:208-215.

[17] Armstrong S.M., Wang C., Tigdi J., Si X., Dumpit C., Charles S., Gamage A., Moraes T.J., Lee W.L., Influenza infects lung microvascular endothelium leading to microvascular leak: role of apoptosis and claudin-5, PloS one. (2012) 7:e47323.

[18] Braniste V., Leveque M., Buisson-Brenac C., Bueno L., Fioramonti J., Houdeau E., Oestradiol decreases colonic permeability through oestrogen receptor beta-mediated up-regulation of occludin and junctional adhesion molecule-A in epithelial cells, The Journal of physiology. (2009) 587:3317-3328.

[19] Phares T.W., Kean R.B., Mikheeva T., Hooper D.C., Regional differences in blood-brain barrier permeability changes and inflammation in the apathogenic clearance of virus from the central nervous system, Journal of immunology. (2006) 176:7666-7675.

[20] Smith J.S., Yager, P.A.., Baer, G.M., A rapid fluorescence focus inhibition test (RFFIT) for determining rabies virus-neutralisng antibody. In: Meslin, F.-X., Kaplan, M,M., Koprowski, H. (Eds.), Laboratory Techniques in Rabies, 4th ed. .pp. 181-192. (1996).

[21] loakeimidis F., Ott C., Kozjak-Pavlovic V., Violitzi F., Rinotas V., Makrinou E., Eliopoulos E., Fasseas C., Kollias G., Douni E., A Splicing Mutation in the Novel Mitochondrial Protein DNAJC11 Causes Motor Neuron Pathology Associated with Cristae Disorganization, and Lymphoid Abnormalities in Mice, PloS one. (2014) 9:e104237.

[22] Zhao L., Toriumi H., Wang H., Kuang Y., Guo X., Morimoto K., Fu Z.F., Expression of MIP-1alpha (CCL3) by a recombinant rabies virus enhances its immunogenicity by inducing innate immunity and recruiting dendritic cells and B cells, Journal of virology. (2010) 84:9642-9648.

[23] Wang H., Zhang G., Wen Y., Yang S., Xia X., Fu Z.F., Intracerebral administration of recombinant rabies virus expressing GM-CSF prevents the development of rabies after infection with street virus, PloS one. (2011) 6:e25414.

[24] Retamal-Diaz A., Riquelme-Neira R., Saez D., Rivera A., Fernandez P., Cabrera A., Guzman C.A., Onate A., Use of S-[2,3-bispalmitoyiloxy-(2R)-propyl]-R-cysteinyl-amido- monomethoxy polyethylene glycol as an adjuvant improved protective immunity associated with a DNA vaccine encoding $\mathrm{Cu}, \mathrm{Zn}$ superoxide dismutase of Brucella abortus in mice, Clinical and vaccine immunology : CVI. (2014) 21:1474-1480.

[25] Singha H., Mallick A.I., Jana C., Isore D.P., Goswami T.K., Srivastava S.K., Azevedo V.A., Chaudhuri P., Owais M., Escheriosomes entrapped DNA vaccine co-expressing Cu-Zn superoxide dismutase and IL18 confers protection against Brucella abortus, Microbes and infection / Institut Pasteur. (2008) 10:10891096. 
[26] Yu D.H., Hu X.D., Cai H., A combined DNA vaccine encoding BCSP31, SOD, and L7/L12 confers high protection against Brucella abortus 2308 by inducing specific CTL responses, DNA and cell biology. (2007) 26:435-443.

\section{Figures}
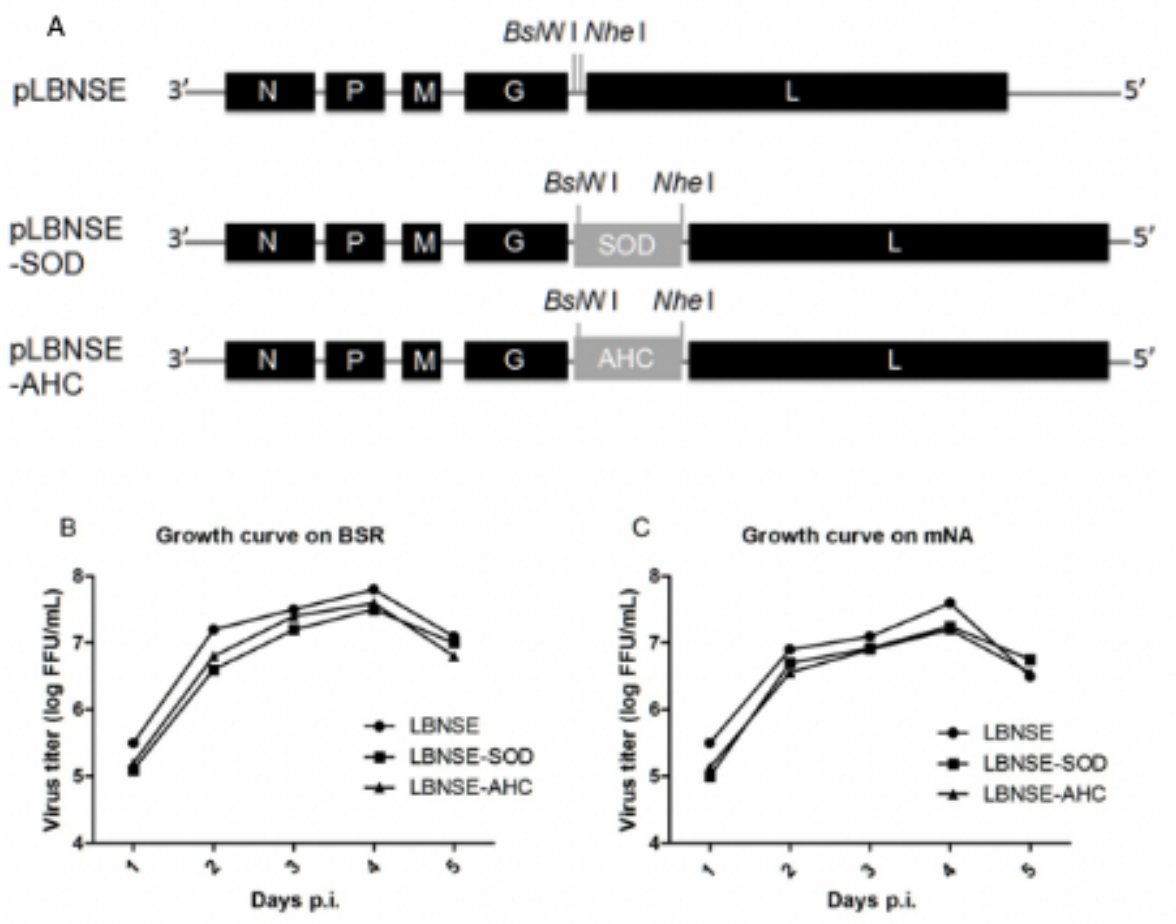

Figure 1

Construction strategy and the growth curve of recombinant virus. A. Schematic diagrams of two fulllength cDNA clones used to rescue recombinant virus. The construction of the individual plasmid, based on pLBNSE, is displayed. Bsiwl and Nhel were introduced ahead of $L$ gene in pLBNSE. The Brucella OMP or AHC gene was inserted by Bsiwl and Nhel. B, C. The growth curve of recombinant vaccine vectors grow in cells. BSR cells (B) or mNA cells (C) were infected with LBNSE, LBNSE-SOD and LBNSE-AHC at a MOI of 0.01 (multicycle growth) or 5 (one-step growth curve). Aliquots of tissue culture supernatants were collected and viral titers were determined in duplicate as indicated. 


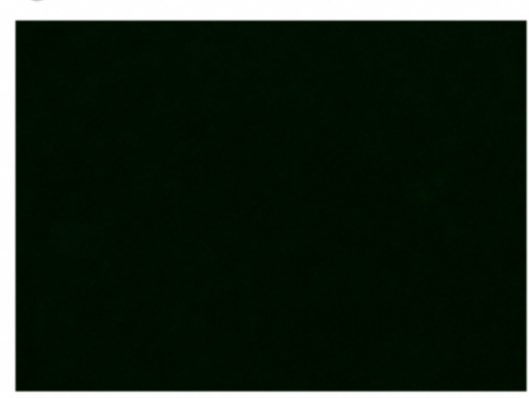

BSR cells

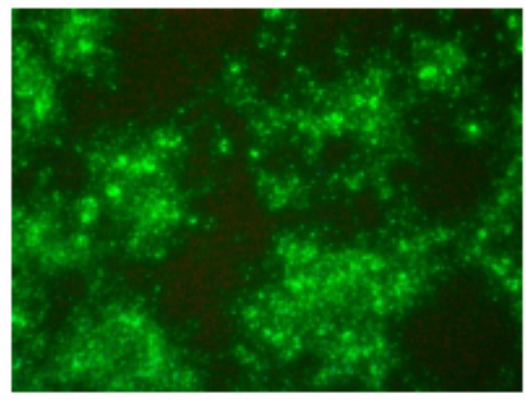

LBNSE-SOD

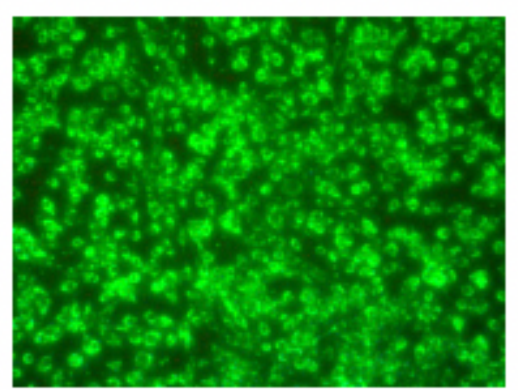

LBNSE

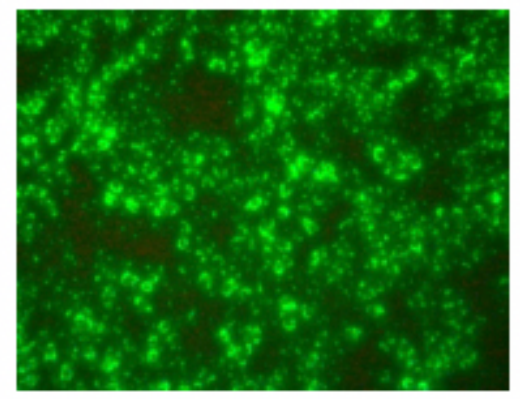

LBNSE-AHC

\section{Figure 2}

Rescue of recombinant rabies viruses. rLBNSE-SOD and rLBNSE-AHC were rescued as described previously. Briefly, the full-length infectious clone, and helper plasmids of N, P, L, G were co-transfected into BSR cells using Lipofectamine 2000 transfection reagent (Grand Island, NY) according to the manufacturer's protocol. After 4 days incubation at $34^{\circ} \mathrm{C}$, the culture medium was discarded and fresh medium replenished for further incubation (3 more days). Cell culture medium was harvested and tested for rescued virus after reaction with FITC-conjugated anti-RABV $\mathrm{N}$ antibody.
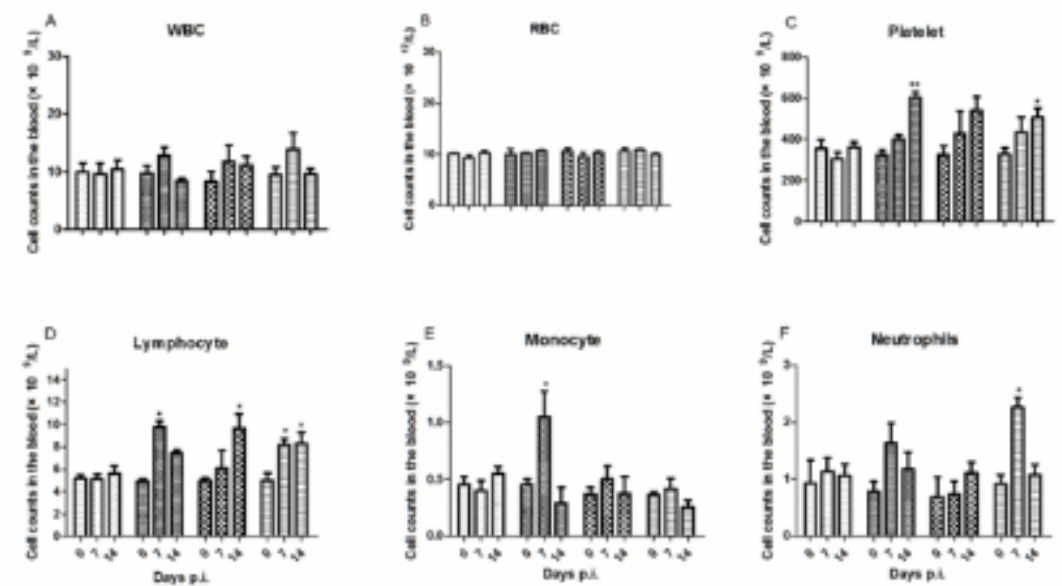

Control

LBNSE

LBNSE-SOD

LBNSE-AHC

\section{Figure 3}

Cells count in the blood of mice. Kunming mice (groups of 9) at the age of 4-6 weeks were treated with Medium and immunized (i.m.) with LBNSE, LBNSE-SOD and LBNSE-AHC at dose of $106 \mathrm{FFU} / \mathrm{mL}$ 
$(100 \mu \mathrm{l} /$ mouse). Mice were euthanized at day 0 , and at day 7, 14 after immunization. Whole blood from each group was collected for hematocyte count: WBC (white blood cell) (A), RBC (red blood cell), (B) Platelet (C), Lymphocyte(D), Monocyte (E), Neutrophils(F). Values represent the average from three animals. Data are expressed as mean values \pm SEM. Asterisks indicate significant differences $\left({ }^{*}, p<0.05\right.$; $\star *, p<0.01$ ) between the corresponding time points of four groups.
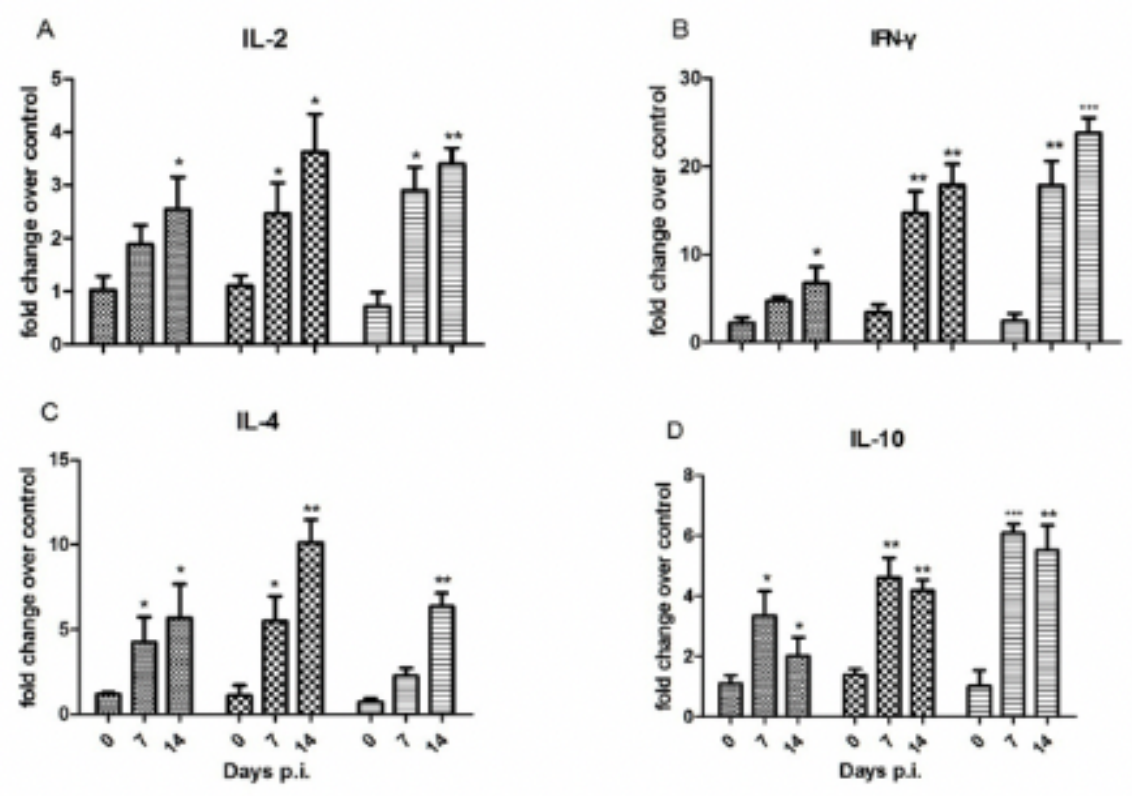

․NSE $\boldsymbol{m}$ LBNSE-SOD $\square$ LBNSE-AHC

\section{Figure 4}

The mRNA level of cytokines detected in the blood of mice. Kunming mice (groups of 9) at the age of 4-6 weeks were treated with Medium and immunized (i.m.) with LBNSE, LBNSE-SOD and LBNSE-AHC at dose of $106 \mathrm{FFU} / \mathrm{mL}(100 \mu \mathrm{l} /$ mouse). Mice were euthanized at day 0 , and at day 7,14 after immunization. Whole blood from each group was collected for the copy number of mRNA level of cytokines measure by real-time PCR: IL-2 (A), IFN- $\gamma(B)$, IL-4(C), IL-10(D). Values represent the average from three animals. Data are expressed as mean values \pm SEM. Asterisks indicate significant differences $(*, p<0.05 ; * *, p<0.01$; $\star \star \star, p<0.001)$ between the corresponding time points of four groups.
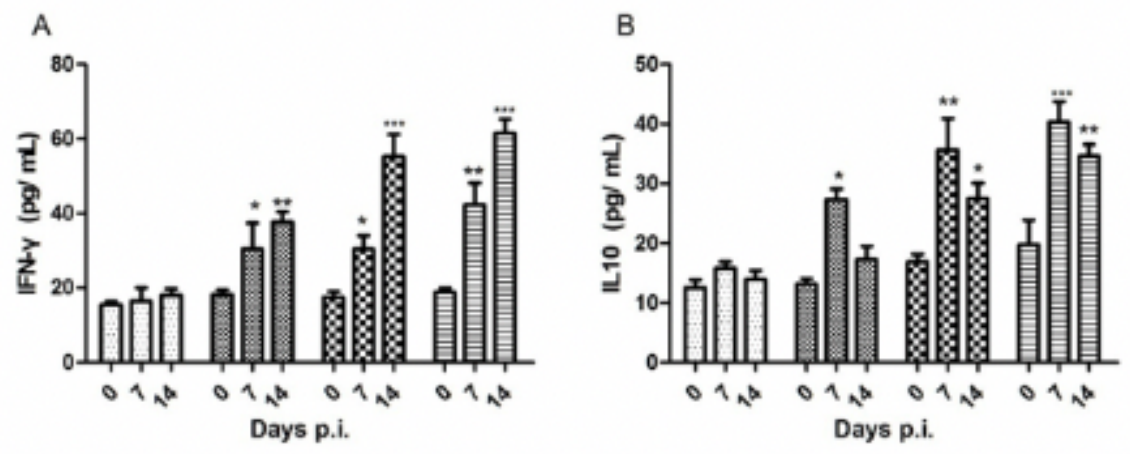


\section{Figure 5}

The ELISA level of cytokines measured in the sera of mice. Kunming mice (groups of 9) at the age of 4-6 weeks were treated with Medium and immunized (i.m.) with LBNSE, LBNSE-SOD and LBNSE-AHC at dose of $106 \mathrm{FFU} / \mathrm{mL}(100 \mu \mathrm{l} / \mathrm{mouse})$. Mice were euthanized at day 0, and at day 7, 14 after immunization. Sera from each group were collected for the ELISA level of cytokines detection by mouse Quantitative ELISA Kit according to the manufacturer's instructions: IFN- $\gamma(A), I L-10$ (B). Values represent the average from three animals. Data are expressed as mean values \pm SEM. Asterisks indicate significant differences $\left({ }^{*}, p<\right.$ $0.05 ; * \star, p<0.01 ; * \star \star, p<0.001)$ between the corresponding time points of four groups.

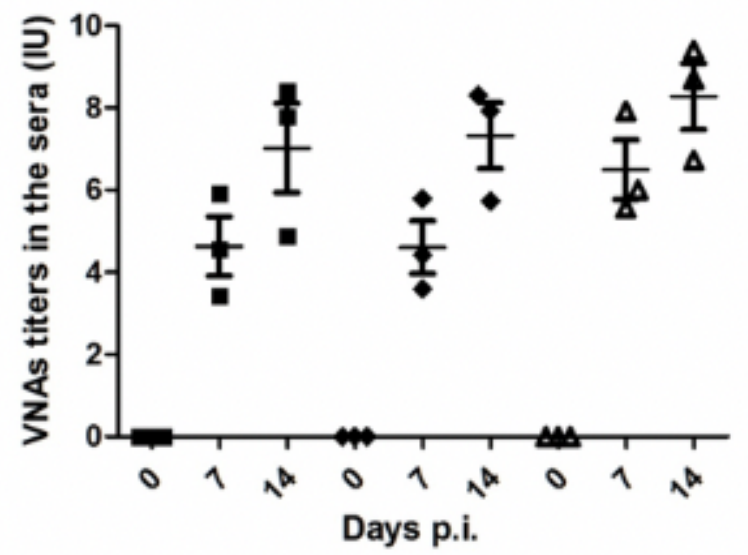

- LBNSE

- LBNSE-SOD

$\Delta$ LBNSE-AHC

\section{Figure 6}

The VNA level against rabies virus in the sera of mice. Kunming mice (groups of 9) at the age of 4-6 weeks were treated with Medium and immunized (i.m.) with LBNSE, LBNSE-SOD and LBNSE-AHC at dose of $106 \mathrm{FFU} / \mathrm{mL}(100 \mu \mathrm{l} / \mathrm{mouse})$. Mice were euthanized at day 0 , and at day 7, 14 after immunization. Sera from each group were collected for the VNA level against rabies virus detection by FAVN assay. Values represent the average from three animals. Data are expressed as mean values \pm SEM. Asterisks indicate significant differences $(*, p<0.05 ; * \star, p<0.01 ; * \star *, p<0.001)$ between the corresponding time points of experimental groups. 


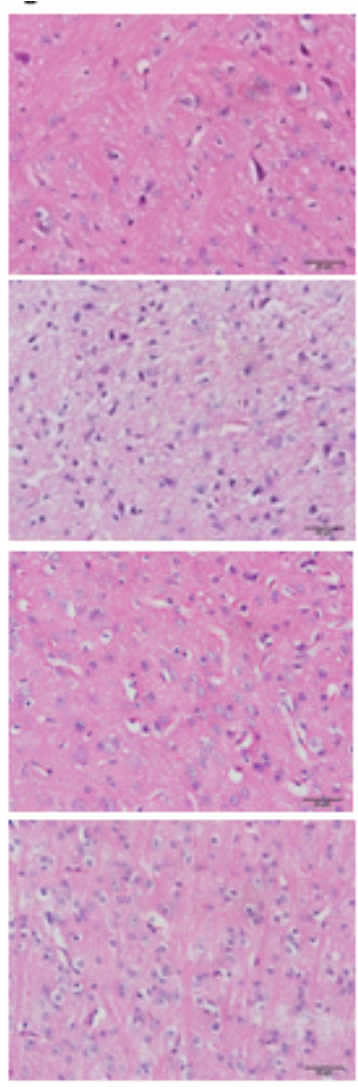

d.p.i 0
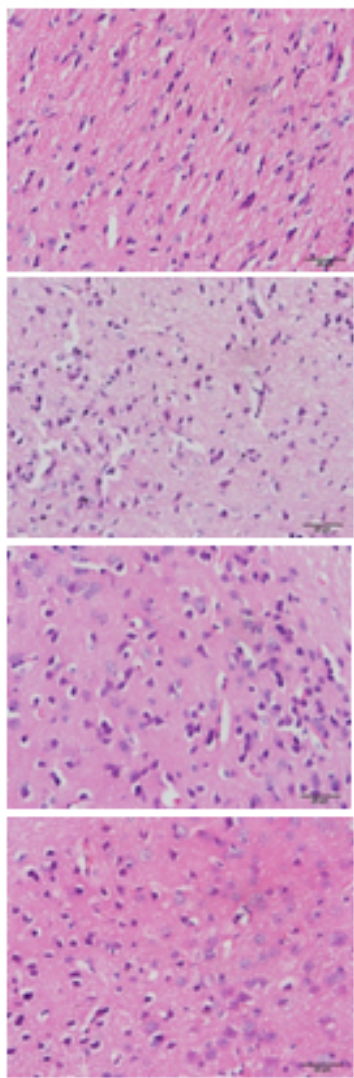

d.p.i 7

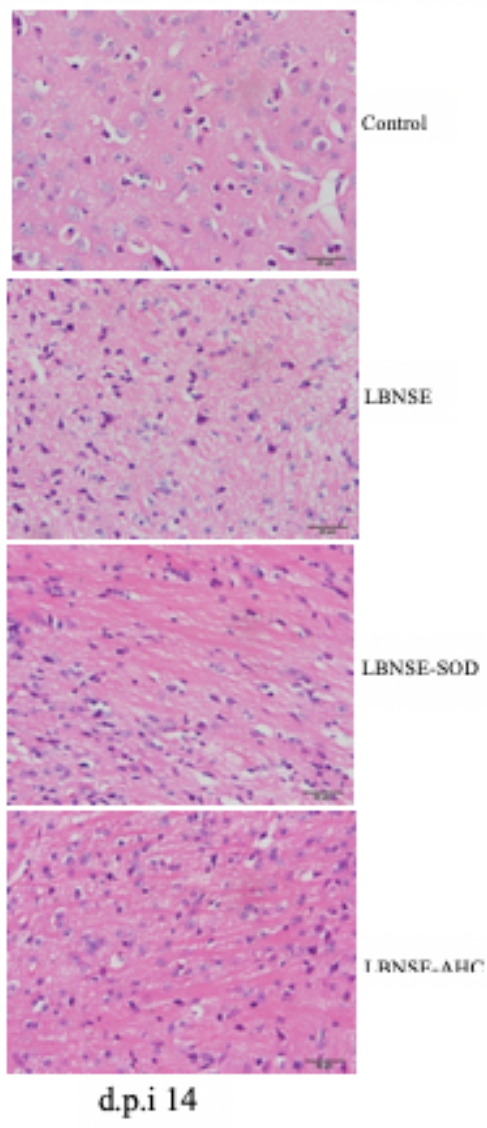

d.p.i 14

\section{Figure 7}

Histopathology observation of the brains in all groups. Kunming mice (groups of 9) at the age of 4-6 weeks were treated with Medium and immunized (i.m.) with LBNSE, LBNSE-SOD and LBNSE-AHC at dose of $106 \mathrm{FFU} / \mathrm{mL}(100 \mu \mathrm{l} / \mathrm{mouse})$. Mice were euthanized at day 0 , and at day 7,14 after immunization. Brain samples from each group were collected for histopathology observation by HE staining.

\section{Supplementary Files}

This is a list of supplementary files associated with this preprint. Click to download.

- NC3RsARRIVEGuidelinesChecklist2014.docx 